\title{
Pyocolpos with imperforate hymen in a 21 days old newborn
}

\author{
Mohammed Khairy Ali ${ }^{1}$, Ali Haron Ali ${ }^{1}$, Ahmed Haress Elsayed ${ }^{1}$, Sherif Abdel-Karem Shazly ${ }^{1}$, Safwat \\ Abdelrady Mohammed ${ }^{1}$
}

Sri Lanka Journal of Obstetrics and Gynaecology 2012; 34: 161-162

\begin{abstract}
Imperforate hymen is one of hymenal variation occurring in as less as 0.0014 to $0.1 \%$ of infants girls. Pyocolpos may be defined as accumulation of an excess of secondary infected cervical secretion in vaginal cavity due to congenital atresia. Female infants with pyocolpos usually present with urological complications or acute gastrointestinal obstruction. We would like to present a rare case of imperforate hymen complicated with pyocolpos in a 21days old female newborn associated with diarrhea and fever. This infant was successfully treated surgically.
\end{abstract}

Key words: Imperforate hymen, pyocolpos

\section{Introduction}

Pyocolpos is accumulation of pus in the vagina. It is a rare complication of hydrocolpos ${ }^{1}$. Hydrocolpos results from congenital vaginal obstruction in combination with maternal hormonal stimulation of

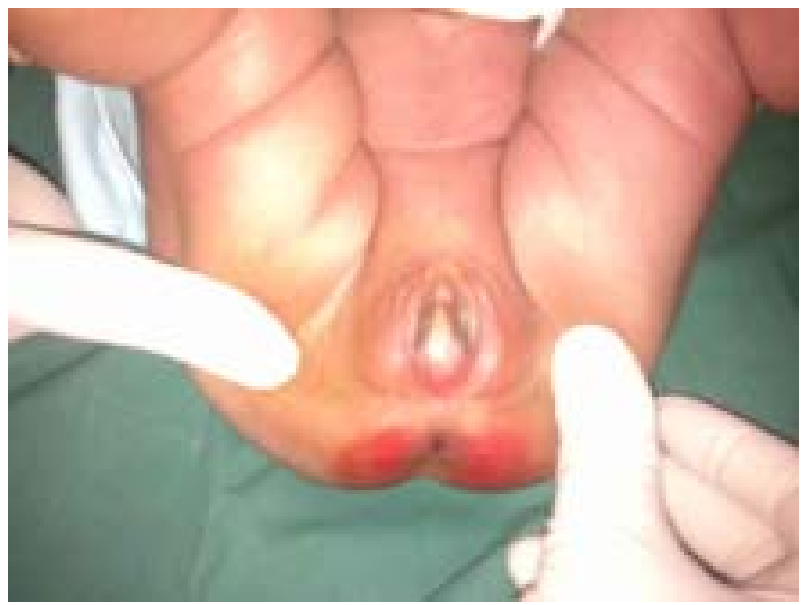

Figure 1. Shows pyocolpos before hymenotomy.

\footnotetext{
${ }^{1}$ Woman's Health Center, Assiut University, Assiut, Egypt. Correspondence: Mohammed Khairy Ali

E-mail:mohammedelkosy@yahoo.com
}

fetal cervical glands. Hydrocolpos usually presents during adolescence and is associated with an imperforate hymen².

\section{Case report}

A 21 days old female newborn was brought to our emergency room because her parents noticed abnormal bulging from the vulva. Her parents said that the newborn had been lethargic and had a poor appetite and diarrhea with intermittent high fever (up to $42^{\circ} \mathrm{C}$ ) of unknown origin for the previous 7 days. She had not had an upper respiratory tract infection. The physical examination revealed no abnormal findings; however, detailed gynecological examination was done under anaesthesia which revealed a bulging imperforate hymen. It was incised by a cruciate incision and $\sim 50 \mathrm{ml}$ of pus-like fluid was drained out. The Figure 1 shows the pyocolpos before hymenotomy and Figure 2 shows pyocolpos after hymenotomy. The fluid was sent for bacterial culture and E. coli and eubacterium species were isolated. Post operative abdominal ultrasonography revealed normal kidney size and parenchyma.



Figure 2: Shows pyocolpos after hymenotomy. 


\section{Discussion}

Pyocolpos is a rare condition ${ }^{3}$. The etiology for the failure to establish patency is not evident. The cause may include an imperforate hymen, a transverse vaginal septum, and maternal estrogen over stimulation. In our case the aetiology was due to imperforate hymen. However, it is often ignored in a genital examination by physicians. Further, if gynecologic abnormality is found, urologic screening studies are recommended to rule out potential associated anomalies. Prenatal diagnosis of imperforate hymen has also been reported as early as 25 weeks gestation. ${ }^{4}$. A thin bulging membrane separating both labia in association with a distended vagina can be seen by ultrasounography. However, there are many interesting points to discuss in this case. First: to our knowledge, this is a first case of 21 days old female with pyocolpos to be documented in Egypt literature. Second: acute intestinal obstruction is reported to be rarely associated with pyocolpos but in our case there was a diarrhea which may be infectious related to pus which accumulated in the vagina.

\section{Conclusion}

Although an imperforate hymen is not a very rare condition in females, the genital examination of newborns, is often ignored by physicians. This case indicates the need to perform a full physical examination, particularly a genital examination, in newborns. Pyocolpos is a rare complication confined to newborn with imperforate hymen.

\section{References}

1. Wall EM, Stone B, Klein BL. Imperforate hymen: a not-sohidden diagnosis. Am J Emerg Med 2003; 21(3): 249-50.

2. Nazir Z, Rizvi RM, Qureshi RN, Khan ZS, Khan Z. Congenital vaginal obstructions: varied presentation and outcome. Pediatr Surg Int 2006; 22(9): 749-53.

3. Graivier L. Hydrocolpos. J Pediatr Surgery 1969; 4: 563-8.

4. Ogunyemi D. Prenatal sonographic diagnosis of bladder outlet obstruction caused by a ureterocele associated with hydrocolpos and imperforate hymen. Am J Perinatol 2001; 18(1): 15-21. 